\title{
The Many Faces of Olex2
}

\section{Horst Puschmann, Oleg Dolomanov}

OlexSy Ltd, Chemistry Department, Durham University, Durham DH1 3LE, UK E-mail: horst.puschmann@gmail.com

Olex2[1] has by now become a firmly established complete package for small-molecule structure determination. 13 years after its first appearance, there are many thousand users of this intuitive packages and the core Olex 2 paper has been cited nearly 5000 times.

Many of our users know and value Olex2 as an easy-to-use interface for various structure solution and refinement packages, notably or course those from the ShelX suite of programs.

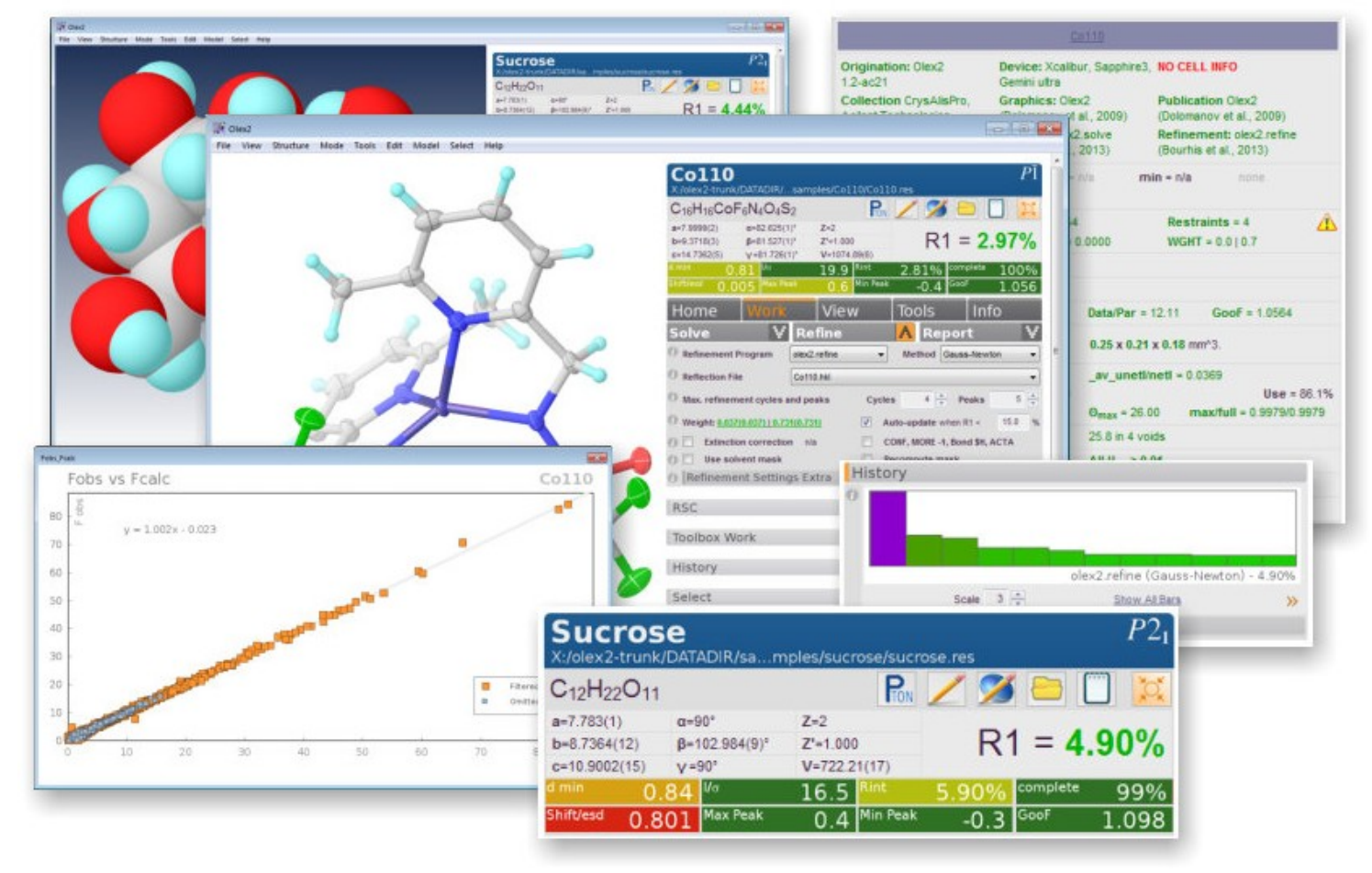

Olex 2 is packed full of features, many of which remain relatively unkown to the normal user. In this presentation will provide an overview overview over the Many Faces of Olex2, concentrating on the features that have been added more recently.

- Everyday Olex2

- Scripting Olex2: From Macros to Python

- The CCTBX, SMTBX[2] and Olex2

- Extension Modules for Olex2

- Scientific Collaborations with Olex2: Hirshfeld Atom Refinement

\section{References}

[1] Dolomanov, O.V.; Bourhis, L.J.; Gildea, R.J.; Howard, J.A.K.; Puschmann, H., OLEX2: A Complete structure solution, refinement and analysis program (2009). J. Appl. Cryst., 42, 339-341.

[2] Bourhis, L.J.; Dolomanov, O.V.; , L.J.; Gildea, R.J.; Howard, J.A.K.; Puschmann, H., "The anatomy of a comprehensive constrained, restrained refinement program for the modern computing environment - Olex2 dissected", Acta Cryst. (2015). A71, pp 59-75. 